\title{
Comparación del método de Thiessen con alternativas más simples mediante simulación de Monte Carlo*
}

\author{
Marcelo Guelfi ${ }^{* *}$ \\ Carlos López-Vázquez ${ }^{* * *}$
}

Recibido el 13 de abril de 2015; aceptado el 16 de agosto de 2015

\begin{abstract}
Estimating the expected value of a function over geographic areas is problem with a long history. In the beginning of the XXth century the most common method was just the arithmetic mean of the field measurements ignoring data location. In 1911, Thiessen introduced a new weighting procedure measuring influence through an area and thus indirectly considering closeness between them. In another context, Quenouville created in 1949 the jackknife method which is used to estimate the bias and the standard deviation. In 1979, Efron invented the bootstrap method which, among other things, is useful to estimate the expected value and the confidence interval (CI) from a population. Although the Thiessen's method has been used for more than 100 years, we were unable to find systematic analysis comparing its efficiency against the simple mean, or even to more recent methods like jackknife or boostrap. In this work we compared four methods to estimate de expected value. Mean, Thiessen, the so called here jackknifed Thiessen and bootstrap. All of them are feasible for routine use in a network of fixed locations. The comparison was made using the Friedman's Test after a Monte Carlo simulation. Two cases were taken for study: one analytic with three arbitrary functions and the other using experimental data from daily rain measured with a satellite. The results show that Thiessen's method is the best estimator in almost all the cases with a $95 \%$ of confidence interval. Unlike the others, the last two considered methods supply a

* Esta es una versión revisada y extendida del trabajo del mismo título presentado en las II Jornadas de Cartografía, 30-31 de octubre de 2014, Montevideo, Uruguay.

** Facultad de Ingeniería, Universidad ORT del Uruguay, correo electrónico: marcelo@mguelfi.com

*** Laboratorio LatinGEO, SGM+ORT, Facultad de Ingeniería, Universidad ORT del Uruguay, correos electrónicos: carloslopez@uni.ort.edu.uy; carlos.lopez@ieee.org
\end{abstract}


suitable CI, but the one obtained through jacknifed Thiessen was more accurate, opening the door for future work.

Key words: Thiessen, Monte Carlo, bootstrap, jackknife.

\section{Resumo}

A estimação do valor esperado de uma função sobre áreas geográficas é um problema que data de tempos atrás. Até o início do século XX o método mais comum consistia em calcular a média aritmética das medidas obtidas em campo, ignorando sua posição geométrica. Em 1911, Thiessen introduziu uma nova fórmula de cálculo que associava cada ponto de medição a um peso relativo a sua área de influência, que levava em conta indiretamente a proximidade entre dados. Em 1949, Quenouville cria, em outro contexto, o método jackknife que é usado para estimar o desvio padrão e a inclinação. Em 1979, Efron inventou o método do bootstrap que, entre outras coisas, é apropriado para estimar o valor esperado de uma população assim como seu intervalo de confiança (IC). Enquanto o método de Thiessen vem sendo usado por mais de um século, não são encontrados estudos sistemáticos que comparem sua eficácia comparado ao método anterior, nem com suas variantes posteriores como jackknife ou bootstrap. Este trabalho consiste na comparação dos quatro métodos de estimação do valor esperado: o do média aritmética, o de Thiessen, o aquí chamado de jackknifed Thiessen e o do bootstrap. Todos eles são adequados para aplicações repetitivas em uma rede de observação fixa. A comparação foi realizada através do Teste de Friedman feita em uma simulação de Monte Carlo. Para os dados são considerados dois casos: um analítico através do estudos de três funções arbitrárias e outro experimental com dados de chuva diária medidos por satélite. Os resultados obtidos mostram que o método Thiessen é o melhor estimador em praticamente todos os casos com nível de confiança de $95 \%$. As últimas duas variantes tem a virtude de fornecer um IC que se mostrou adequado, embora o jackknifed Thiessen tenha resultados mais precisos, abrindo assim a porta para futuras investigações.

Palavras chave: Thiessen, Monte Carlo, bootstrap, jackknife.

\section{Resumen}

La estimación del valor esperado de una función sobre áreas geográficas es un problema que data de tiempo atrás. Hasta principios del siglo XX el método más común solía ser calcular la media aritmética de las medidas obtenidas en el campo, ignorando su posición geométrica. En 1911, Thiessen introdujo una nueva forma de cálculo que asignaba a cada punto de medición un peso relativo al área de influencia, que tenía en cuenta indirectamente la proximidad entre datos. En 1949, Quenouville crea, en otro contexto, el método de jackknife que se utiliza para 
estimar el sesgo y la desviación estándar. En 1979, Efron inventa el método de bootstrap que, entre otras cosas, es apropiado para estimar el valor esperado de una población así como su intervalo de confianza (IC). Si bien el método de Thiessen lleva usándose hace más de un siglo, no se han encontrado estudios sistemáticos que comparen su eficacia frente al método anterior ni frente a variantes posteriores como jackknife o bootstrap. Este trabajo consiste en comparar cuatro métodos para la estimación del valor esperado: el de la media aritmética, el de Thiessen, el aquí denominado jackknifed Thiessen y el de bootstrap. Todos ellos son aptos para aplicaciones repetitivas en una red de observación fija. La comparación se realizó mediante el Test de Friedman tras una simulación de Monte Carlo. Para los datos se consideran dos casos: uno analítico mediante el estudio de tres funciones arbitrarias, y otro experimental con datos de lluvia diaria medidos por satélite. Los resultados obtenidos muestran que el método Thiessen es el mejor estimador en prácticamente todos los casos con el $95 \%$ de nivel de confianza. Las últimas dos variantes tienen la virtud de suministrar un IC que se mostró adecuado, aunque jackknifed Thiessen resultó mucho más ajustado, abriendo así la puerta para futuras investigaciones.

Palabras clave: Thiessen, Monte Carlo, bootstrap, jackknife.

\section{Introducción}

Tanto en las ciencias de la Tierra como en otras áreas, es necesario en ocasiones calcular un valor representativo de una variable en un cierto dominio. Como ejemplo puede citarse la densidad de población, la lluvia promedio en una cuenca, etc. Para aplicaciones en geociencias es corriente que se den ciertas situaciones:

- El dato a observar se conoce solamente en unos pocos lugares (puntos)

- Es costoso (o en ocasiones imposible) agregar puntos en posiciones arbitrarias

- Los usos dan más importancia al valor promedio que al dato individual

Éstas y otras circunstancias aplican al caso de datos meteorológicos. La red de estaciones de medición ha sido tradicionalmente fija, y por lo tanto se registran series temporales en puntos precisos. Una variable muy popular es la lluvia diaria; ella participa en cálculos hidrológicos, climáticos, agronómicos, etc., y es comunicada regularmente en anuarios estadísticos para caracterizar las condiciones de una zona. La motivación para este trabajo está asociada al cálculo de su valor esperado. Sin perjuicio de ello, hay aplicaciones indirectas. El estándar NSSDA de exactitud posicional (Federal Geographic Data Committee - FGDC, 1998) describe cómo calcular un número representativo del error posicional de una cartografía. Ese número resulta de la expresión: 


$$
\text { Exactitud }=2.4477 * 0.5 *\left(R E M C_{x}+R E M C_{y}\right)
$$

Cada uno de los dos sumandos del final responde a la fórmula genérica:

$$
\left(\operatorname{REMC}_{x}\right)^{2}=\frac{\sum_{i=1}^{N}\left(x_{i}-x_{\text {exact }, i}\right)^{2}}{N}
$$

lo cual a su vez es el promedio de la discrepancia al cuadrado entre la coordenada del punto de control y el valor declarado como exacto para ese punto. Las coordenadas del punto no participan en la expresión sino a través de la discrepancia. Esta expresión del promedio es exactamente la misma que se utilizaba en forma rutinaria antes del trabajo de Thiessen (1911) para variables meteorológicas como la lluvia diaria. ¿Puede hacerse algo mejor con el NSSDA? Como se verá más abajo, el método de Thiessen fue creado para dar mejores estimaciones de magnitudes asociadas con expresiones de este tipo.

Desde que existen redes de observación meteorológica se ha intentado caracterizar la variabilidad espacial de las mediciones. Las redes se diseñan teniendo en consideración esos aspectos (distancia mutua, valores estimados de la correlación espacial, etc.), así como restricciones prácticas (facilidad de acceso, disponibilidad de energía, etc.). A principios del siglo Xx la capacidad de cálculo era limitada, y por lo tanto la estimación de la "lluvia representativa" se hacía con el promedio simple de los datos disponibles, independientemente de la posición y la distancia mutua. Ya en 1911 Thiessen (Thiessen, 1911) reconocía que ese procedimiento tendía a sesgar los resultados, especialmente cuando la densidad de las estaciones variaba mucho en el territorio. Si hay varias estaciones relativamente próximas entre sí, el valor recogido en ellas incide mucho en el promedio espacial cuando en realidad representan un fenómeno local. Por ello propuso el método hoy conocido como de los polígonos de Thiessen. El mismo se basa en calcular la media ponderada de los valores disponibles en las $\mathrm{N}$ estaciones. El factor de ponderación es proporcional al área de la región circundante que contiene puntos más próximos a esa estación que a cualquier otra. Geométricamente la misma se construye por segmentos de mediatrices mutuas entre las estaciones disponibles. La Figura 1 muestra las regiones en un caso sencillo.

El criterio de Thiessen se deriva en alguna medida de la primera Ley de Tobler (Tobler, 1970) que se expresa como: "todas las cosas están relacionadas entre sí, pero las cosas más próximas en el espacio tienen una relación mayor que las distantes". Thiessen adopta como criterio de proximidad la distancia geométrica, en una aplicación literal de lo que explicitará Tobler sesenta años después. 


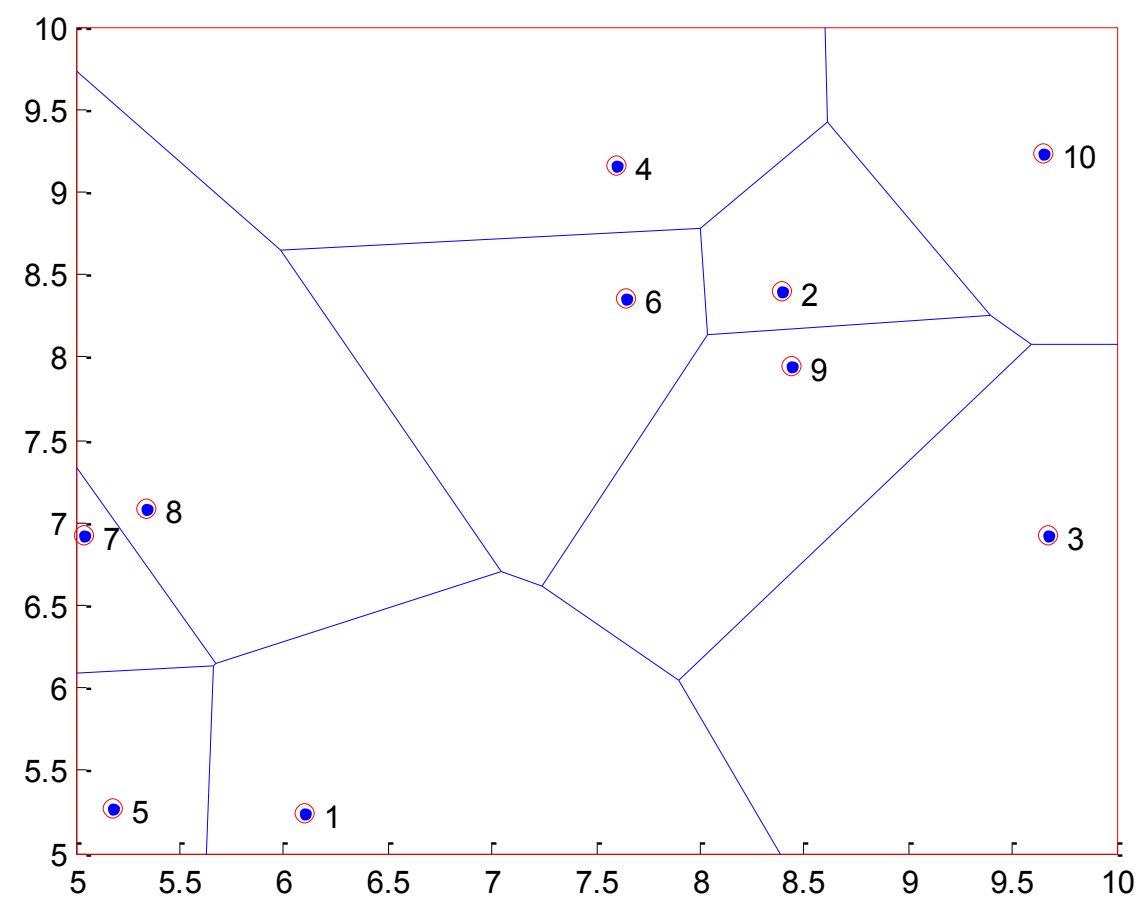

Figura 1. Ilustración de los polígonos de Thiessen para el caso $\mathrm{N}=10$. Fuente: Elaboración propia.

A pesar de su formulación simple, el cálculo de los polígonos ha sido un tema de gran interés, tanto por parte de la comunidad de las geociencias como por matemáticos e informáticos. Fuera del área meteorológica el problema se conoce como Diagrama de Voronoi, y existe incluso una serie de congresos específicos dedicados al tema (<http://bioinf.spbau.ru/isvd2013/home $>$ ) que atiende problemas como nuevos métodos de cálculo, implementación en computadores paralelos, nuevas aplicaciones, etc.

La razón del persistente interés en el tema está asociada a que el cálculo no es trivial, y para muchas dimensiones o muchos puntos se requieren cuidados especiales. Teniendo esto en cuenta, cabe preguntarse si realmente el esfuerzo extra de cálculo se justifica atendiendo la calidad de la estimación resultante. Contra lo que podría suponerse, no se han encontrados trabajos básicos que comparen el desempeño de otras alternativas para la estimación de la misma magnitud. Es por ello que surge la idea de este trabajo, intentando confirmar (o desmentir) que el método de Thiessen es superior a otros, y que a pesar de su mayor complejidad está 


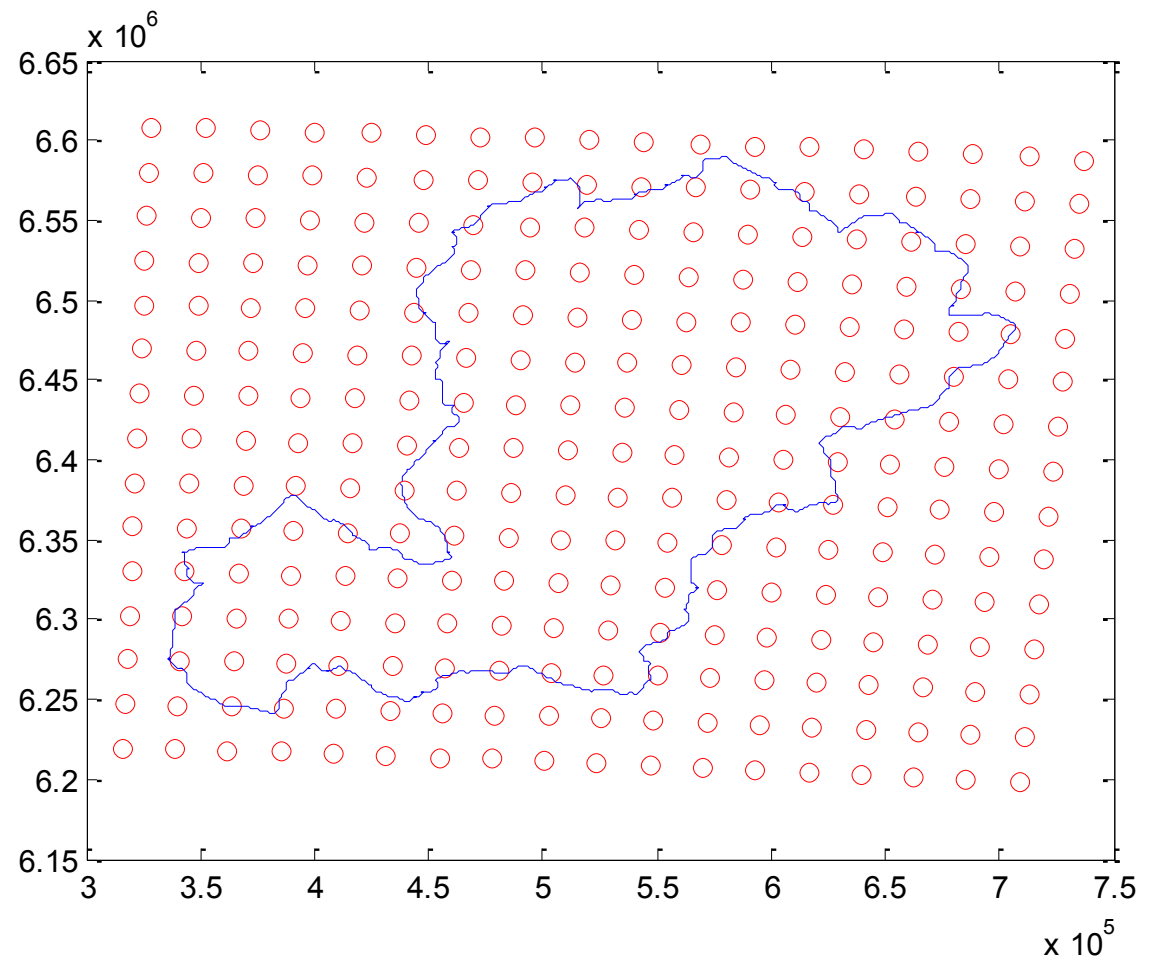

Figura 2. Cuenca de la represa del Rincón del Bonete y localización del centro de los pixeles.

Fuente: Elaboración propia.

justificado su uso por su mejor desempeño. Para realizar la comparación debe recurrirse a un experimento controlado (que se describirá más adelante) y a la aplicación de test estadísticos que den certeza matemática a las conclusiones. Para esto último se recurrirá al Test de Friedman (Friedman, 1937; 1939). El mismo permite caracterizar varios métodos que han sido ensayados en diferentes eventos y tras ello puede establecerse un orden relativo entre ellos. Tras especificar un nivel de confianza (usualmente 95\%) el test permite concluir si uno de los métodos es sistemáticamente mejor que los demás. En la situación que se analizará, los métodos se ordenarán de acuerdo a la proximidad entre su estimación y el verdadero valor.

El Test de Friedman requiere un número mínimo de métodos y eventos. Para llegar a ese mínimo de métodos se han considerado las siguientes posibilidades: 1) media aritmética de los datos disponibles 2) media remuestreada (bootstrapped) 3) polígonos de Thiessen y 4) jackknifed Thiessen. El número de eventos se decide como parte de la simulación de Monte Carlo. Este artículo está organizado de la 
siguiente forma: tras la introducción, se describirán los antecedentes y métodos utilizados para el análisis. Luego se mostrarán los datos obtenidos y finalmente se presentarán las conclusiones.

\section{Antecedentes metodológicos}

\section{Método de Thiessen}

En 1911 Alfred Thiessen propuso un método alternativo para el cálculo del promedio de lluvias en grandes superficies. Dada el área de estudio y la localización de las estaciones meteorológicas, tomó en cuenta el área de influencia de dichas estaciones y ponderó cada valor por dicha área. Este proceso dio origen a lo que se conoce como Teselación de Thiessen, o también Diagrama de Voronoi. Este método ha sido utilizado por más de cien años en problemas relativos a la lluvia, variables meteorológicas en general, y un sinnúmero de otras aplicaciones en áreas diversas.

Si bien Thiessen no manejó ese concepto, lo que se está intentando estimar está estrechamente ligado a la integral de la lluvia en el espacio. Para el cálculo numérico de integrales es habitual que se intente sustituir primero la función por un interpolante, y luego se calcula la integral de este último. El motivo es práctico: el interpolante suele ser más simple que la función original, y por lo tanto los cálculos demorarán menos. En el problema práctico bajo estudio la función no es conocida, por lo que sólo está disponible el interpolante (que no es único). El estimador de Thiessen es la integral exacta de uno particular de ellos, denominado Vecino más próximo. Este interpolante es discontinuo en los bordes, y constante dentro de cada una de las regiones, adoptando el valor del punto dato en ellas. Por resultar de la intersección de semiplanos, puede demostrarse que las regiones son convexas.

\section{Método de jackknifed Thiessen}

El método de jackknife fue propuesto originalmente por Quenouille (1949) para el tratamiento de series temporales y se popularizó rápidamente. García-Guzmán y Calatrava-Requena (1978) presentan un resumen general del método y analizan sus ventajas y desventajas. El método consiste en un remuestreo sin reemplazo de la población disponible, en el cual se obtienen $\mathrm{N}$ valores del estimador deseado tras aplicarlo a $\mathrm{N}$ conjuntos de $\mathrm{N}-1$ elementos en que falta el i-ésimo dato. Tras ello (y en este trabajo) se calcula la mediana de esos estimadores, que pasará a ser lo que aquí se denominará jackknifed Thiessen, y sus valores máximos y mínimos, los que serán adoptados como estimadores de los extremos del intervalo de confianza (IC) de la forma $(\min +\max ) / 2+2 *(\max -\min ) *[-1,1]$. Los números "max" y "min" se corresponden al valor máximo y mínimo del estimador de Thiessen calculado con 
cada conjunto de $\mathrm{N}$ remuestreos del evento. En muchas aplicaciones el número $\mathrm{N}$ es bajo (pocas decenas) o medio (hasta el centenar), por lo que el tiempo de cálculo requerido para el jackknifed Thiessen puede no ser significativo. Esta técnica puede aplicarse fácilmente si ya está implementado el método de Thiessen, no requiriendo desarrollar mucho código adicional.

\section{Método de Bootstrap}

Efron (1979) presenta este método como una alternativa al jackknife tradicional. A diferencia del método anterior, propone hacer un remuestreo con reemplazo, y luego construir el estimador como promedio de los obtenidos en cada remuestreo. También del experimento pueden derivarse intervalos de confianza para ese valor. El remuestreo con reemplazo significa que un dato puede estar repetido (incluso más de una vez) en la remuestra a la que luego se le calculará el promedio. Desde el punto de vista computacional, es más intensivo que el mero promedio pero sustancialmente menos demandante que el de Thiessen y jackknifed Thiessen. El código es relativamente sencillo, y sólo requiere la existencia de una biblioteca para generar números seudoaleatorios.

\section{Datos y métodos}

\section{Descripción del problema para el caso analítico}

En el caso analítico se parte de una función conocida de la que es posible saber con exactitud el valor de la integral a estimar. Mediante una simulación de Monte Carlo se hacen $\mathrm{M}$ cálculos en los que se eligen cada vez $\mathrm{N}$ puntos al azar dentro del dominio. En esas localizaciones se evalúa el valor de la función y finalmente se calcula la media aritmética, el bootstrap, Thiessen y finalmente el jackknifed Thiessen. El resultado se presenta como una tabla de $\mathrm{M}$ filas y 4 columnas que permite comparar qué tan buenas son las aproximaciones por cada método en relación al valor exacto, creando un ranking entre ellos.

Para el caso analítico se utilizaron las siguientes tres funciones:

$$
\begin{aligned}
& f(x, y)=x^{2} \cdot y \\
& f(x, y)=\sin (x) \cdot \sin (3 y) \\
& f(x, y)=\text { morrisonIII }(1000 x, 1000 y)
\end{aligned}
$$

Las primeras dos son arbitrarias. La tercera es debida a Morrison (1971) y consiste en una serie trigonométrica de 48 términos truncada hasta el tercer armónico. La misma fue obtenida como el ajuste a datos experimentales de una localización real, por lo que se estima representativa de una topografía. En los tres casos el dominio de integración es el cuadrado [0 1] x [0 1]. Si bien es accesible el 
valor analítico, para cada función se calculó numéricamente la integral en el dominio con un error relativo de $10^{-6}$.

\section{Descripción del problema para el caso experimental}

En el segundo estudio se parte de una imagen de satélite con valores de lluvia diaria, obtenida de $<$ http://disc.sci.gsfc.nasa.gov/precipitation/tovas > A los efectos del problema bajo estudio puede considerarse a la imagen como una tabla que posee $\mathrm{P}>>\mathrm{N}$ puntos (pixeles) observados en campo. Igual que antes, para hacer la comparación se simula $\mathrm{M}$ veces la elección de $\mathrm{N}$ de esos puntos y se repiten los cálculos. A diferencia del caso previo ahora no hay valor "exacto" analítico disponible. Se adopta como valor exacto al resultante del cálculo con Thiessen utilizando todos los pixeles disponibles.

Se usó la imagen correspondiente a la ventana [-34.3 -30.5] de latitud y [-52.5 -48] longitud del día 20 de junio de 2014 que incluye íntegramente a la cuenca de la represa de Rincón del Bonete (Uruguay). El contorno de la cuenca y la localización del centro de los 270 pixeles se muestran en la Figura 2. Tras aplicar Thiessen con los 270 valores registrados, el valor promedio en la cuenca para ese día resultó ser igual a $10.8 \mathrm{~mm} /$ día, el cual se adoptó como valor exacto. Para la simulación se utilizaron únicamente los 97 puntos interiores al borde de la cuenca (polígono en azul) de Figura 2, los cuales no son una muestra sino que constituyen el universo del experimento.

\section{Organización de los cálculos}

Sea $\mathrm{N}$ el número de puntos con datos conocidos. Se utilizaron distintos valores de $\mathrm{N}$ para determinar cuánto mejora la estimación del verdadero valor a medida que $\mathrm{N}$ aumenta. Se analizaron para $\mathrm{N}$ los valores 5, 10, 20, 40 y 50. Para cada función y N, se realizó una simulación de Monte Carlo. En cada uno de ellos y para el caso analítico, las coordenadas de los $\mathrm{N}$ puntos fueron generadas en forma pseudoaleatoria, especificando la semilla de forma hacer repetible la secuencia con las mismas condiciones. Con esas coordenadas se evaluó la función analítica, obteniéndose los $\mathrm{N}$ valores funcionales. Con ellos, y para cada evento, se obtiene una estimación del valor esperado mediante la aplicación de los cuatro métodos mencionados. Para el caso experimental se seleccionaron al azar los $\mathrm{N}$ puntos de entre los disponibles, reteniendo su valor y sus coordenadas.

La comparación entre métodos se realizó siguiendo el Test de Friedman (Friedman, 1937; 1939) el cual consiste en analizar la hipótesis de que los métodos no se diferencian entre sí. El dato de entrada es una tabla del ranking entre métodos, con tantas columnas como métodos y tantas filas como eventos. Cada elemento es un número ordinal $(1,2,3$, etc.) que indica el desempeño relativo de cada método. 
El test admite la posibilidad de empates, tanto en los datos de entrada como en los resultados. En el caso bajo análisis el desempeño fue calculado tras comparar la diferencia en valor absoluto entre el resultado numérico y el verdadero valor. El método calificado como 1 sería aquel que para ese evento discrepa menos (en valor absoluto) y el calificado como 4 el que discrepa más. Para la aplicación del test se especificó un nivel de confianza de 95\%.

La simulación de Monte Carlos inició con $\mathrm{M}=2000$. Se generaron 2 grupos disjuntos y se comparó el resultado del Test de Friedman que dio distinto para cada grupo. Se procedió a ir incrementando cada grupo con 2,000 eventos y compararlos en nuevamente. Al llegar a 10,000 eventos, el resultado del Test fue igual para cada grupo, evitando las posibles implicancias de los números seudoaleatorios generados. Para graficar los resultados se usó el total de 20,000 eventos. La máquina utilizada para los cálculos fue del tipo Intel, con procesador i7 4770K (4 cores $3.5 \mathrm{GHz}$ ) y la simulación del total de casos requirió 96 horas.

\section{Resultados obtenidos}

A continuación se muestran los datos obtenidos de las simulaciones de cada función. Las filas (de arriba hacia abajo) representan los $\mathrm{N}$ puntos obtenidos al azar, en el orden 5, 10, 20, 40, y 50. Las columnas (de izquierda a derecha) muestran los resultados para cada método analizado: media aritmética, bootstrap, Thiessen y jackknifed Thiessen.

Nótese en la Figura 3 la fuerte dispersión que muestra la media aritmética independientemente de $\mathrm{N}$, y la rápida concentración que se logra con jackknifed Thiessen. Nótese también que los estimadores tienden a ser sesgados cuando $\mathrm{N}$ crece. Se observa que el IC es progresivamente más estrecho, y que las distribuciones experimentales no lucen en general como de Gauss. En la Figura 4 se repite el patrón de concentración alrededor del verdadero valor del jackknifed Thiessen en comparación con los otros, pero ahora no es tan notorio el sesgo. En este ejemplo (tal vez más realista que el previo) las distribuciones tienden a ser simétricas. En el caso de la función de Morrison III (Morrison, 1971) ilustrado en la Figura 5 se muestra que los métodos derivados de Thiessen son relativamente insesgados, y que el jackknifed Thiessen confirma su tendencia de tener los intervalos de confianza más ajustados. El verdadero valor en ningún caso (ningún método y ningún $\mathrm{N}$ ) estuvo fuera del IC al 90\%, aunque para IC estrechos estuvo próximo al techo. Para el caso experimental, en la Figura 6 se nota que el comportamiento de los métodos es similar al de las funciones analíticas, dando una mejor estimación de jackknifed Thiessen y un sesgo decreciente cuando $\mathrm{N}$ aumenta. La dispersión muestra una distribución bastante simétrica, asimilable a una de Gauss. 

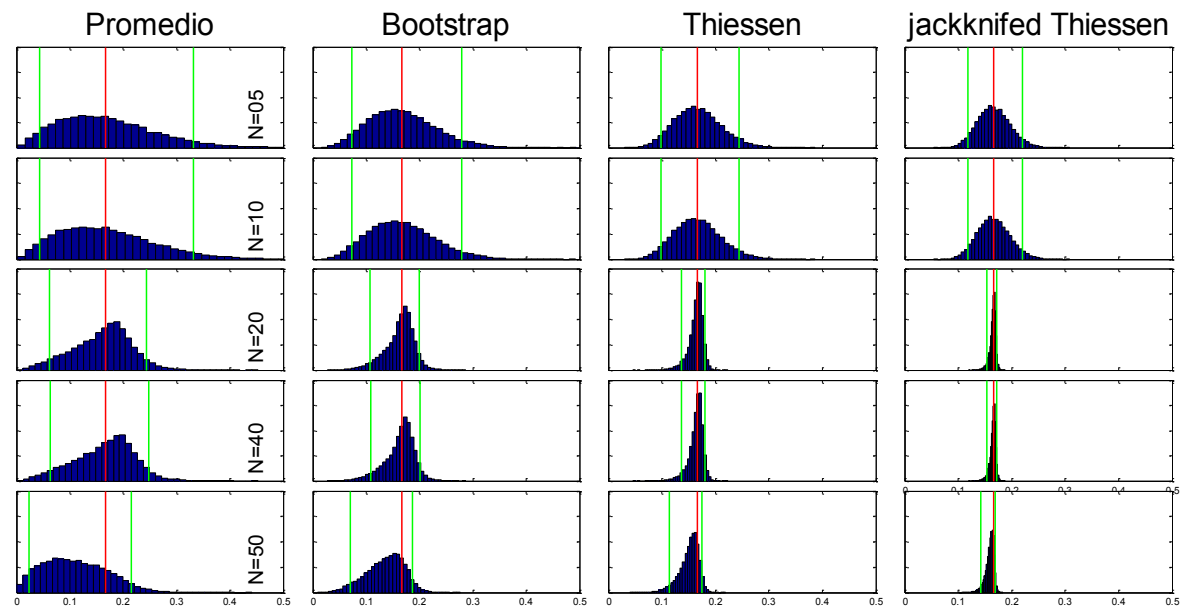

Figura 3. Resultados de simulación para la función $\mathrm{x} 2 * \mathrm{y}$. En rojo se indica el verdadero valor (idéntico en todas las gráficas), y en verde los percentiles experimentales al 5 y 95\%. El rango de abscisas es idéntico en todos los gráficos.

Fuente: Elaboración propia.
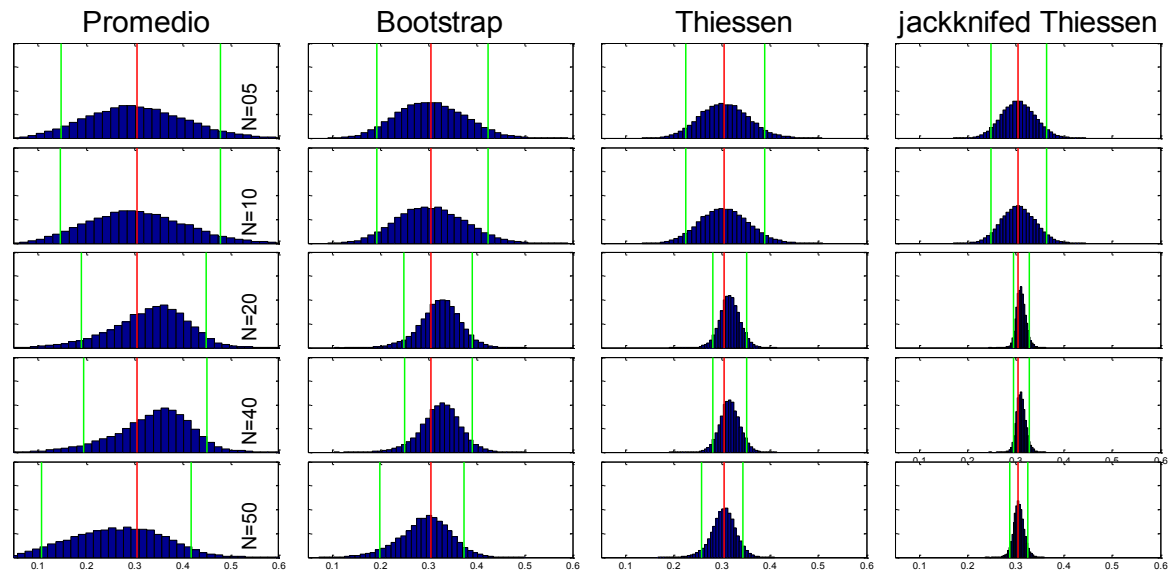

Figura 4. Resultados de simulación para la función de $\sin (\mathrm{x}) * \cos (3 \mathrm{y})$. En rojo se indica el verdadero valor (idéntico en todas las gráficas), y en verde los percentiles experimentales al 5 y $95 \%$. El rango de abscisas es idéntico en todos los gráficos.

Fuente: Elaboración propia. 

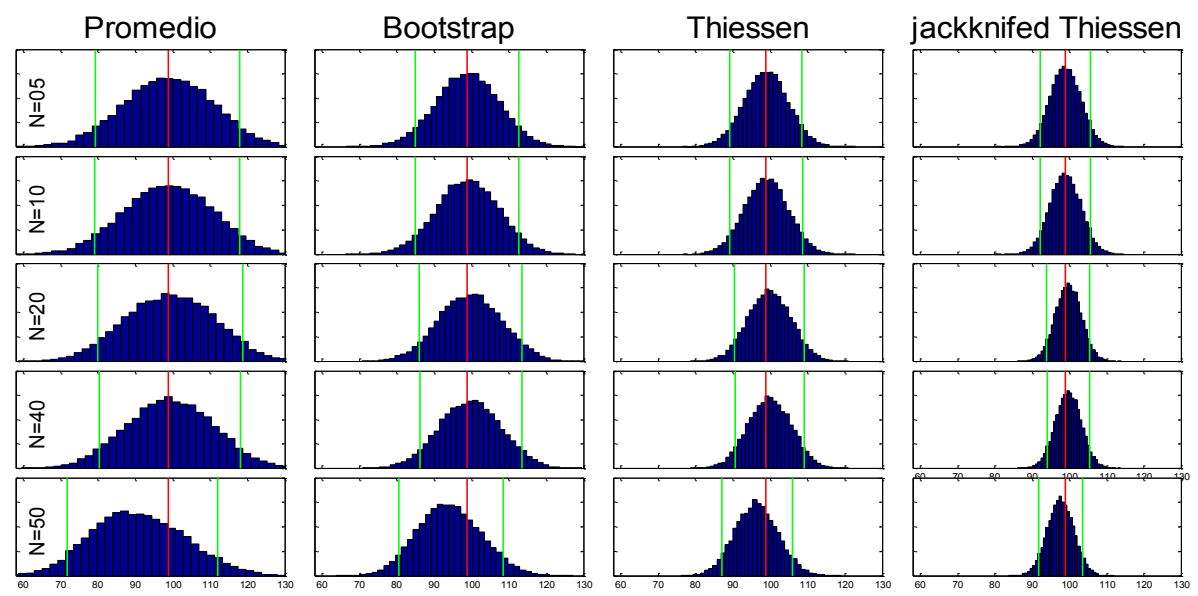

Figura 5. Resultados de simulación para la función de Morrison III. En rojo se indica el verdadero valor (idéntico en todas las gráficas), y en verde los percentiles experimentales al 5 y $95 \%$. El rango de abscisas es idéntico en todos los gráficos.

Fuente: Elaboración propia.
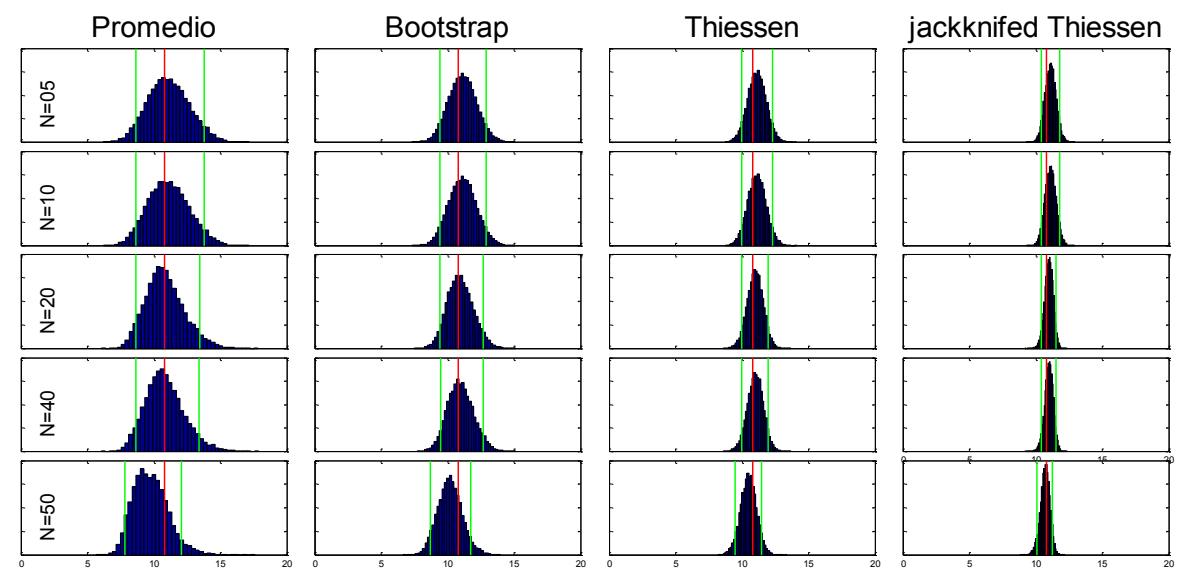

Figura 6. Resultados de simulación para estimar la lluvia en la región de la Figura 2. En rojo se indica el verdadero valor (idéntico en todas las gráficas), y en verde los percentiles experimentales al 5 y $95 \%$. El rango de abscisas es idéntico en todos los gráficos.

Fuente: Elaboración propia. 
Puede observarse que los métodos han quedado ordenados, de izquierda a derecha, por la longitud decreciente del IC, lo que muestra un favoritismo por los dos últimos. Sin embargo, debe señalarse que ese resultado no sería decisivo, ya que lo representado es consecuencia de una simulación. En un caso práctico (un único evento) debería valorarse positivamente que el bootstrap y el jackknifed Thiessen generan ellos mismos un IC para ese evento. En los cálculos realizados (no mostrados aquí) se pudo ver que en más del 90\% de los casos el IC definido como [media- $2 *$ desviación, media $+2 *$ desviación] para el bootstrap efectivamente contenía el valor exacto cuando $\mathrm{N}=10,20,40$ o 50 , mientras que para el caso de $\mathrm{N}=5$ el mismo intervalo lo incluía en más del $84 \%$ de los casos. Por su parte en el IC de jackknifed Thiessen incluía al valor exacto en más de un $92 \%$ de los casos.

De todas formas, los gráficos previos aún no son decisivos para responder a la pregunta de "¿vale la pena el esfuerzo de programar Thiessen y sus variantes, frente a alternativas más simples?" porque se han utilizado histogramas y se pierde la información dentro de cada evento. Para ello puede utilizarse el test de Friedman. En la Tabla 1 puede observarse el ranking entre los cuatro métodos considerados, para las tres funciones y en función del valor de N. El valor de "1" indica que fue el mejor, y el valor de "4" el peor. Si estadísticamente no puede discernirse que un método es mejor que otro ello se indica como un empate: ver por ejemplo el caso de la función Morrison III para $\mathrm{N}=40$, que arroja un empate entre el tercer y cuarto método. $\mathrm{Si}$, como era el objetivo de este trabajo, la pregunta era relativa a la comparación entre el mero promedio y el método de Thiessen, la conclusión para todas las funciones, excepto Morrison III, es que sistemáticamente Thiessen es más adecuado. Para la función de Morrison III esa precedencia falla cuando $\mathrm{N}=5$, 10 o 20, mientras que para $\mathrm{N}$ mayores el método de Thiessen supera al mero promedio.

Tabla 1

Resultados del Test de Friedman para las tres funciones analíticas consideradas

\begin{tabular}{|c|c|c|c|c|c|c|c|c|c|c|c|c|c|c|c|c|}
\hline \multirow{2}{*}{$\frac{N}{5}$} & \multicolumn{4}{|c|}{$x^{2} y$} & \multicolumn{4}{|c|}{$\operatorname{sen}(x) * \operatorname{sen}(3 y)$} & \multicolumn{4}{|c|}{ Morrison III } & \multicolumn{4}{|c|}{ Lluvia } \\
\hline & 3 & 4 & 1 & 2 & 3 & 4 & 1 & 2 & 2 & 3 & 4 & 1 & 3 & 4 & 2 & 1 \\
\hline 10 & 3 & 4 & 1 & 2 & 3 & 4 & 2 & 1 & 2 & 3 & 4 & 1 & 3 & 4 & 1 & 2 \\
\hline 20 & 3 & 4 & 2 & 1 & 3 & 4 & 2 & 1 & 2 & 2 & 4 & 1 & 3 & 3 & 1 & 2 \\
\hline 40 & 3 & 4 & 2 & 1 & 3 & 4 & 2 & 1 & 3 & 3 & 2 & 1 & 3 & 4 & 1 & 2 \\
\hline 50 & 3 & 4 & 2 & 1 & 3 & 4 & 2 & 1 & 3 & 4 & 2 & 1 & 3 & 4 & 1 & 2 \\
\hline
\end{tabular}

Fuente: Elaboración propia. 
Lo inesperado de este experimento viene por el desempeño del jackknifed Thiessen: es prácticamente en todos los casos la mejor opción, excepto cuando el número de puntos es muy pequeño, o como en el caso de la lluvia que únicamente queda en segundo lugar cuando $\mathrm{N}=10$. Posiblemente ese resultado sea estadísticamente cuestionable dado el pequeño tamaño de la muestra. El método de bootstrap aplicado para el cálculo de la media dio resultados comparables con el promedio, aunque típicamente lo supera. La ventaja esencial respecto al mero promedio es la posibilidad de suministrar un IC, calculable incluso en situaciones prácticas, por lo que sería recomendable considerar su uso. Comparte esa ventaja con el jackknifed Thiessen.

El ranking fue construido tras considerar 20,000 eventos con un nivel de confianza de $95 \%$.

\section{Conclusiones}

A partir del análisis de los histogramas y de los resultados del Test de Friedman obtenidos de las simulaciones, puede concluirse que en todas las funciones estudiadas, salvo en la superficie de Morrison III y para $\mathrm{N}$ pequeños, el método de Thiessen ofrece sistemáticamente una mejor estimación que la media aritmética y su variante el bootstrap. Tras la simulación realizada, ese resultado puede ahora apoyarse en un nivel de confianza del 95\%, y de acuerdo con la literatura consultada se entiende que éste es un resultado original. Para la función Morrison III en particular, se precisa un $\mathrm{N}$ igual o superior a 40. Una explicación de este comportamiento es la gran variación que tiene la superficie en $(\mathrm{x}, \mathrm{y})$. Con un valor pequeño de $\mathrm{N}$ el método de Thiessen no logra capturar esas variaciones.

Por su parte, jackknifed Thiessen resulta ser un mejor estimador que el propio Thiessen en las funciones analíticas. Esta mejora es a base de tiempo extra de cálculo, que se hace significativo cuando $\mathrm{N}$ aumenta. Para las funciones analíticas el tiempo fue $1.3 \mathrm{x}$ con $\mathrm{N}=5,2.8 \mathrm{x}$ con $\mathrm{N}=10$ y $4 \mathrm{x}$ con $\mathrm{N}=20$. Dada la diferencia significativa de tiempos, podría pensarse si vale la pena el cálculo. No obstante, una ventaja que ofrece frente a Thiessen es que además del valor produce un IC. Si el problema lo justifica, una alternativa a explorar es la de realizar este cálculo mediante el uso de cores de tarjetas gráficas.

Para las funciones analizadas bootstrap resulta en casi todos los casos peor que la media, salvo algún caso particular donde empata. Pero al igual que jackknifed Thiessen, este método brinda un IC. Más allá de estos casos particulares, bootstrap es un método conocido y comprobado en su efectividad, económico y sencillo y por lo tanto de uso práctico si se requiere un IC. 


\section{Bibliografía}

Efron, B. (1979). "Bootstrap Methods: Another Look at the Jackknife", The Annals of Statistics, 7(1), 1-26.

FGDC, 1998. "Geospatial Positioning Accuracy Standards Part 3: National Standard for Spatial Data Accuracy", Federal Geographic Data Committee, FGDC-STD007.3-1998, 28 pp.

Friedman, M. (1937). "The use of ranks to avoid the assumption of normality implicit in the analysis of variance", Journal of the American Statistical Association (American Statistical Association), 32(200), 675-701.

Friedman, M. (1939). "A correction: The use of ranks to avoid the assumption of normality implicit in the analysis of variance", Journal of the American Statistical Association (American Statistical Association), 34(205), 109.

García-Guzmán, A. y Calatrava-Requena, J. (1978). “Algunas consideraciones sobre la naturaleza de la técnica Jackknife de estimación y las ventajas e inconvenientes de su uso en diversos problemas de inferencia estadística", Estadística Española, 78-79, 57-73.

Morrison, J.L., (1971). "Method-Produced Error in Isarithmic Mapping”, American Congress on Surveying and Mapping. Technical Monograph No. CA-5, 75 pp.

Quenouille, M.H. (1949). "Approximate tests of correlation in time series", Journal of the Royal Statistical Society, Series B, 11, 18-44.

Thiessen, A. (1911). "Precipitation averages for large areas", Monthly Weather Review, 39(7), 1082-1084

Tobler, W.R. (1970). "A computer movie simulating urban growth in the Detroit region", Economic Geography, 46, 234-40. 